\title{
A resolved outflow of matter from a brown dwarf
}

\author{
Emma T. Whelan ${ }^{1}$, Thomas P. Ray ${ }^{1}$, Francesca Bacciotti ${ }^{2}$, Antonella Natta ${ }^{2}$, Leonardo Testi ${ }^{2}$ \\ $\&$ Sofia Randich ${ }^{2}$
}

The birth of stars involves not only accretion but also, counterintuitively, the expulsion of matter in the form of highly supersonic outflows ${ }^{1,2}$. Although this phenomenon has been seen in young stars, a fundamental question is whether it also occurs among newborn brown dwarfs: these are the so-called 'failed stars', with masses between stars and planets, that never manage to reach temperatures high enough for normal hydrogen fusion to occur $^{3}$. Recently, evidence for accretion in young brown dwarfs has mounted $^{4-6}$, and their spectra show lines that are suggestive of outflows $^{7-9}$. Here we report spectro-astrometric data that spatially resolve an outflow from a brown dwarf. The outflow's characteristics appear similar to, but on a smaller scale than, outflows from normal young stars. This result suggests that the outflow mechanism is universal, and perhaps relevant even to the formation of planets.

The nearby $\rho$ Ophiuchi cloud (at a distance of 125 pc; ref. 10) is an excellent example of a stellar nursery. It was as part of a near-infrared survey of this region ${ }^{11}$ that $\rho$ Oph 102 was first detected. Its mass is given as $60 M_{\mathrm{J}}$ (ref. 7), where $1 M_{\mathrm{J}}$ equals the mass of Jupiter or 0.001 solar masses $\left(0.001 M_{\odot}\right)$. This places it firmly within the brown dwarf mass range $\left(0.013 M_{\odot} \leq M_{\mathrm{BD}} \leq 0.075 M_{\odot}\right)^{3}$, and indeed this object has been spectroscopically confirmed to be a brown dwarf? . There is also strong evidence for the presence of an accretion disk $^{4}$ and, in fact, the accretion rate (derived from its $\mathrm{H} \alpha$ profile) ${ }^{7}$ is estimated to be approximately $10^{-9} \mathrm{M}_{\odot} \mathrm{yr}^{-1}$. Finally, it has been noted that the spectrum of $\rho$ Oph 102 contains a number of forbidden emission lines, suggesting an outflow ${ }^{7}$.

Tracing an outflow from a young star is done using a variety of techniques depending upon wavelength. When the star itself is sufficiently evolved to be optically visible-that is, much of the surrounding natal gas and dust has been driven away-the outflow can be followed almost right back to the star through its permitted and forbidden line emission ${ }^{1}$. Nearest the source, the light from the collimated outflow is most intense, giving rise to the so-called 'micro-jets' observed, for example, from $\mathrm{T}$ Tauri stars, the precursors to stars like our Sun ${ }^{12}$. In extreme cases, the outflow is observed only very close $\left(\leq 1^{\prime \prime}\right)$ to the source ${ }^{13}$ : that is, within the typical 'seeing' disk for ground-based telescopes. It is under such circumstances that the technique of spectroastrometry comes to the fore (see Methods and Supplementary Information).

Our high resolution echelle observations (see Methods and Fig. 1) of $\rho$ Oph 102 show its forbidden emission lines; for example, [O I] at wavelengths of 6,300 and $6,363 \AA$ and [S II] at 6,731 $\AA$, are moderately blueshifted (radial velocity $V_{\mathrm{r}} \approx-45 \mathrm{~km}^{-1}$ ). Such radial velocities are very similar to those seen in outflows from classical T Tauri stars, and might be expected of an outflow from a young brown dwarf, as its escape velocity is similar ${ }^{14}$. We note also the absence of a redshifted outflow component. This is again typical of T Tauri stars, and is interpreted in terms of an obscuring disk that hides the redshifted component from our view ${ }^{13}$. We can thus immediately infer the presence of a disk around this brown dwarf (see below for a limit on its size). Such a conclusion is also in line with the mid-infrared excess seen from this object ${ }^{4}$. (Note that all velocities are with respect to the systemic velocity of the brown dwarf. The latter was derived from the Li photospheric absorption line at $6,708 \AA$, and equals $7 \pm 8 \mathrm{~km} \mathrm{~s}^{-1}$ in the Local Standard of Rest frame.)

Another indication that an outflow is present comes from a cursory examination of the $\mathrm{H} \alpha$ line (Fig. 2). Its profile is clearly asymmetrical, that is, the blueshifted wing of the line appears to be absorbed in a P Cygni-like fashion. We note however that we do not observe a classical P Cygni profile, that is, one that dips below the continuum. Such a profile is in any event a rare occurrence even amongst $\mathrm{T}$ Tauri stars ${ }^{15}$.

If we are dealing with a scaled-down version of the outflow phenomenon seen in $\mathrm{T}$ Tauri stars, then we expect the centre of emission in forbidden lines to be spatially offset from the continuum, that is, the brown dwarf. This offset is due to the fact that, for a collimated outflow, such lines are quenched close to the star once the electron density becomes high enough ${ }^{13}$. In the case of outflows from $\mathrm{T}$ Tauri stars, typical offsets of $30-75 \mathrm{AU}\left(0.2^{\prime \prime}-0.5^{\prime \prime}\right.$ at $\left.150 \mathrm{pc}\right)$ are seen, for example, in the [O I] doublet at 6,300 and $6,363 \AA$ and the [S II] doublet at 6,717 and 6,731 $\AA$ (ref. 13). If we assume that brown dwarf outflows have similar opening angles and velocities to those from $\mathrm{T}$ Tauri stars, then the point at which the critical density is reached may naively be taken to scale with $\dot{M}_{\text {jet }}^{1 / 2}$, where $\dot{M}_{\text {jet }}$ is the jet's mass loss rate (see Supplementary Information). Assuming that the latter depends linearly on the accretion rate, we would expect typical spatial offsets to be 3-10 times smaller in brown dwarf outflows in comparison to those from T Tauri stars.

Spectro-astrometric (emission centroid offset versus velocity) plots are shown in Fig. 1 for the [OI] doublet, $\mathrm{H} \alpha$ and the [S II] line at $6,731 \AA$. Because of high electron densities close to the brown dwarf, the $[\mathrm{S} I \mathrm{I}]$ line at $6,717 \AA$ was too weak to provide a usable spectro-astrometric signal. Here the spatial offsets were measured after continuum subtraction (see Methods and Supplementary Information for details).

We now consider the main results from the various offset versus velocity plots. First, the centroids of all the measurable forbidden lines are displaced to the south, that is, they have negative offsets with respect to the continuum. These offsets reach a maximum of $0.08^{\prime \prime}-0.1^{\prime \prime}$ at a blueshifted velocity of about $-40 \mathrm{~km} \mathrm{~s}^{-1}$. We have already noted the absence of any corresponding redshifted emission and that this suggests the presence of an obscuring disk. The scale of the blueshifted offset would imply a minimum (projected) disk radius of $0.1^{\prime \prime}$ ( $\geq 15 \mathrm{AU}$ at the distance of the $\rho$ Ophiuchi cloud) in order to hide any redshifted component.

Second, there is no clear spatial offset in $\mathrm{H} \alpha$, even though its higher signal to noise ratio potentially allows us to measure even smaller offsets than observed in the forbidden lines. This is in agreement with the idea that most of the $\mathrm{H} \alpha$ emission arises from accretion $^{7}$ on much smaller scales than are being probed here. It is 

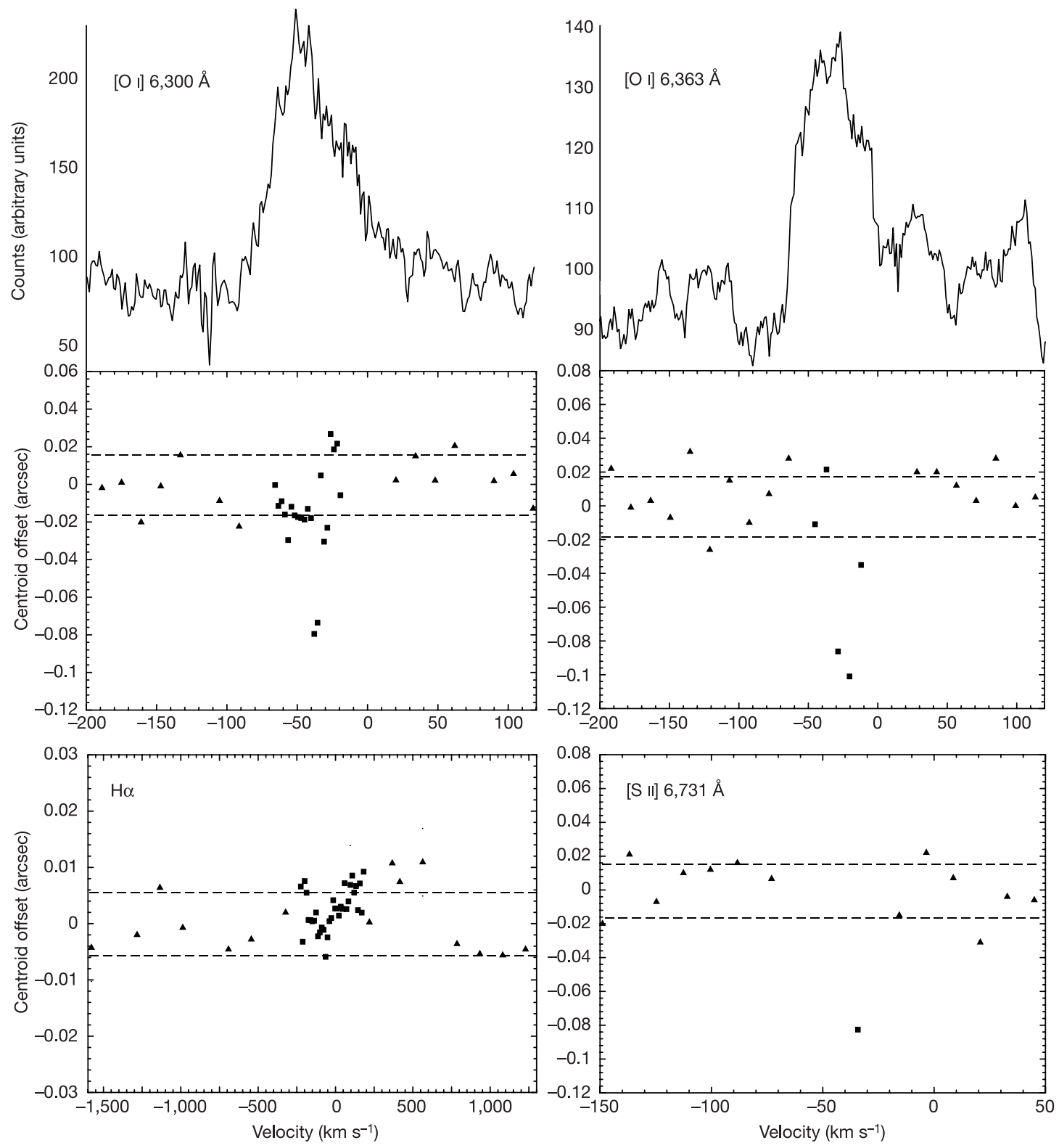

Figure 1 Resolving the outflow from $\rho$ Oph 102. Line profiles (top row) and spectro-astrometric plots (middle row) for the [O I] doublet at 6,300 and $6,363 \AA$, and spectro-astrometric plots (bottom row) for the $\mathrm{H} \alpha$ and the $6,731 \AA$ line of $\left[\mathrm{S}_{\mathrm{II}}\right]$. The [O I] night sky lines at $-9 \mathrm{~km} \mathrm{~s}^{-1}$ are subtracted. No offset measurements were made in their vicinity (thus accounting for the data point gaps). Continuum and line offset points are

also worth pointing out that if, as is almost certainly the case, the blue-ward dip in the $\mathrm{H} \alpha$ profile is caused by P Cygni-like absorption from an outflowing wind in front of the star, no offset should be expected.

Third, both the line profiles and the spectro-astrometric signatures are very similar (albeit on somewhat smaller scales) to what is seen in $\mathrm{T}$ Tauri stars with 'micro-jets'. In particular, the observed velocities and offsets in the various forbidden lines are within the range we would expect for a collimated outflow from a brown dwarf (see also Supplementary Information). We suggest that direct imaging (using, for example, Fabry-Perot systems) of this and other candidate brown dwarf outflows should now be attempted. Such observations will, however, be very challenging, even with large telescopes, because of the expected faintness of the outflow. represented by triangles and squares, respectively. Velocities are systemic, and offsets are in the north-south direction with negative offsets to the south. Dashed lines delineate the $\pm 1 \sigma$ error envelope. For $\mathrm{H} \alpha$, note the much smaller offset scale. The $\left[\mathrm{S}_{\mathrm{II}}\right]$ line is blueshifted to around $-40 \mathrm{~km} \mathrm{~s}^{-1}$.

\section{METHODS}

Spectro-astrometry. Conceptually the principles of spectro-astrometry are easy to understand. The profile of a star is smeared by atmospheric turbulence to appear gaussian (at least to a first approximation) rather than point-like. Whereas the width of the profile is determined by the so-called seeing, how accurately we can determine the centroid of emission is, in theory for fixed seeing, limited only by the strength of the observed signal to noise ratio. Increasing the total number of detected photons increases the positional, or astrometric, accuracy, so that, in principle, milliarcsecond precision is possible with very large ground based telescopes ${ }^{16-18}$.

Consider now a long-slit spectrum of a close binary system consisting of two virtually identical stars. We will assume that the slit is orientated along the same position angle as the binary. (We note that strictly this is not necessary: it is only necessary that the slit is not orthogonal.) If the separation of the binary is considerably less than the seeing, the profile of the system in the spatial direction 


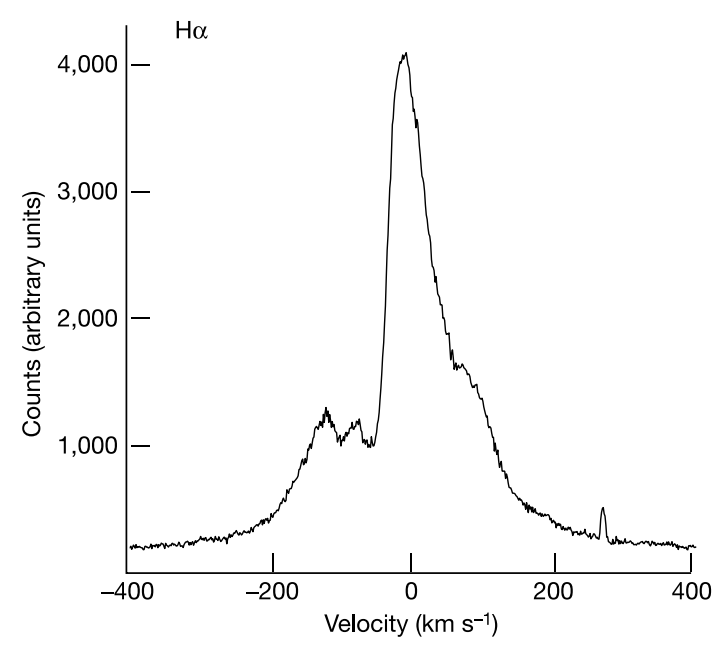

Figure 2 | The outflow signature in the $\mathrm{H} \alpha$ profile of $\rho$ Oph 102. The $\mathrm{P}$ Cygni-like dip in the line profile is a strong signature of outflow activity. $\mathrm{H} \alpha$ emission from the brown dwarf is absorbed as it passes through material moving outwards along our line of sight. Because this material is moving towards us, the dip is on the blueward side of the line. Classical T Tauri stars are strong $\mathrm{H} \alpha$ emitters, and P Cygni $\mathrm{H} \alpha$ profiles originally confirmed that such protostars drive outflows. The dip in the $\mathrm{H} \alpha$ emission of $\rho$ Oph 102 is at approximately the outflow radial velocity determined from the forbidden lines.

will consist of a single gaussian - that is, the system is unresolved and the centroid of emission will lie exactly between the two components. Now suppose that one of the two stars differs slightly from the other in being a strong $\mathrm{H} \alpha$ emitter; in such a case, the emission centroid will shift towards that star in the spectrum at the position of the $\mathrm{H} \alpha$ line. In this way it is possible to resolve certain types of binaries with separations well within the seeing $\operatorname{limit}^{19}$. In the case of a jet (pure emission line region) plus star (continuum source), one can go further and interpolate the continuum across a line, thereby allowing its contribution to be removed. It is then possible to measure separately the spatial centroid of the pure emission line region and determine its offset with respect to the continuum, that is, the parent star. Moreover, as the line can be emitted over a range of wavelengths, owing to the Doppler effect, it may also be feasible to recover spatio-kinematic information. For example, if the jet is bipolar, that is, it has oppositely directed blue- and redshifted flows from the source; the emission centroid of the red and blue wings of the line will be displaced to opposite sides of the continuum centre.

The detailed method by which we measure offsets can briefly be described as follows. First, the centroid of the continuum emission in the spatial direction is determined using a one-dimensional gaussian fit. The line of such centroids, in the dispersion direction but excluding any region where emission lines are present, is then fitted with a second-order polynomial, over a range of typically 200-300 $\AA$. In this way, instrumental curvature and tilting, with a characteristic frequency many times larger than the width of any line, is determined. The fit, to the centre of the continuum, is then subtracted from the actual measured centroids, leaving residuals that are evenly scattered about the abscissa (that is, the fit defines the zero offset line). The continuum data points shown in Fig. 1 are thus the residuals. Finally, the two-dimensional fit to the continuum, broadened to take account of the point spread function, is subtracted from the emission lines. Any emission line offsets are then measured.

The accuracy (in arcseconds) of the method is set by the error in the centroid of the gaussian fit, which depends on the seeing and the number of detected photons, $N$. Formally, the error is given by Seeing/ $\left[2(2 \ln 2)^{1 / 2} \mathrm{~N}^{1 / 2}\right]$, assuming that photon noise is the only source of noise. $N$, of course, is a function of the binning and the spatial sampling (pixel width). This explains why, for example, we can achieve a higher spectro-astrometric accuracy with a bright line, such as $\mathrm{H} \alpha$, than a weak one, for example, the [S II] line at 6,731 $\AA$. In some cases, it is necessary to bin up a weak line in the dispersion direction, as we have done to varying degrees for the $\left[\mathrm{O}_{\mathrm{I}}\right]$ doublet and the $\left[\mathrm{S}_{\mathrm{II}}\right]$ line at $6,731 \AA$, to achieve sufficient signal to noise ratio. Note that we sometimes use different binning factors for the continuum, in comparison with the line, so as to achieve a similar signal to noise ratio in both. This allows us to have comparable offset errors in both components, and to define the common $1 \sigma$ error lines shown in Fig. 1. As can be seen from the plots, the typical limiting offset that we can measure in the spatial direction $(3 \sigma)$ is around 30 mas. This corresponds to $4.5 \mathrm{AU}$ at the distance to the $\rho$ Ophiuchi cloud.

Echelle spectroscopy. The high resolution spectra of $\rho$ Oph 102 were taken with the UV-visual Echelle Spectrograph (UVES) on the European Southern Observatory's $8 \mathrm{~m}$ Kueyen Telescope, one of the telescopes in the Very Large Telescope (VLT) suite, in May 2003. A total of three $45 \mathrm{~min}$ exposures of the target were made, together with a series of flats and biases as well as an observation of an arc lamp for wavelength calibration. The slit was orientated north-south and had a width of $1^{\prime \prime}$ while the seeing was $0.65^{\prime \prime}$. The central wavelength was set at $580 \mathrm{~nm}$, giving a spectral range of $450-680 \mathrm{~nm}$. Only the red part of the spectrum from 580 to $680 \mathrm{~nm}$, however, was analysed. The pixel scale was $0.182^{\prime \prime}$ and the spectral resolution $R=40,000$. The data were reduced using standard Image Reduction and Analysis Facility (IRAF) routines.

\section{Received 23 September 2004; accepted 23 March 2005.}

1. Eislöffel, J., Mundt, R., Ray, T. P. \& Rodríguez, L. F. in Protostars and Planets IV (eds Mannings, V., Boss, A. P. \& Russell, S. S.) 815-840 (University of Arizona Press, Tucson, 2000).

2. Königl, A. \& Pudritz, R. E. in Protostars and Planets IV (eds Mannings, V., Boss, A. P. \& Russell, S. S.) 759-787 (University of Arizona Press, Tucson, 2000).

3. Basri, G. Observations of brown dwarfs. Annu. Rev. Astron. Astrophys. 38, 485-519 (2000).

4. Natta, A. et al. Exploring brown dwarf disks in $\rho$ Ophiuchi. Astron. Astrophys. 393, 597-609 (2002).

5. Pascucci, I., Apai, D., Henning, T. \& Dullemond, C. P. The first detailed look at a brown dwarf disk. Astrophys. J. 590, L111-L114 (2003).

6. Jayawardhana, R., Mohanty, S. \& Basri, G. Evidence for a T Tauri phase in young brown dwarfs. Astrophys. J. 592, 282-287 (2003).

7. Natta, A. et al. Accretion in brown dwarfs: An infrared view. Astron. Astrophys. 424, 603-612 (2004)

8. Comerón, F., Fernández, M., Baraffe, I., Neuhäuser, R. \& Kaas, A. A. New lowmass members of the Lupus 3 Dark Cloud: Further indications of pre-main sequence evolution strongly affected by accretion. Astron. Astrophys. 406, 1001-1017 (2003).

9. Fernández, M. \& Comerón, F. Intense accretion and mass loss of a very low mass young stellar object. Astron. Astrophys. 380, 264-276 (2001).

10. de Geus, E. J., de Zeeuw, P. T. \& Lub, J. Physical parameters of stars in the Scorpio-Centaurus OB Association. Astron. Astrophys. 216, 44-61 (1989).

11. Greene, T.P. \& Young, E. T. Near-infrared observations of young stellar objects in the $\rho$ Ophiuchi Dark Cloud. Astrophys. J. 395, 516-528 (1992).

12. Dougados, C., Cabrit, S., Lavalley, C. \& Ménard, F. T Tauri star microjets resolved by adaptive optics. Astron. Astrophys. 357, L61-L64 (2000).

13. Hirth, G. A., Mundt, R. \& Solf, J. Spatial and kinematic properties of the forbidden emission line region of T Tauri stars. Astron. Astrophys. Suppl. 126, 437-469 (1997).

14. Masciadri, E. \& Raga, A. C. Looking for outflows from brown dwarfs. Astrophys. J. 615, 850-854 (2004).

15. Muzerolle, J., Calvet, N. \& Hartmann, L. Emission-line diagnostics of T Tauri magnetospheric accretion. II. Improved model tests and insights into accretion physics. Astrophys. J. 550, 944-961 (2001).

16. Takami, M., Bailey, J., Gledhill, T. M., Chrysostomou, A. \& Hough, J. H. Circumstellar structure of RU Lupi down to AU scales. Mon. Not. R. Astron. Soc 323, 177-187 (2001).

17. Whelan, E. T., Ray, T. P. \& Davis, C. J. Paschen beta emission as a tracer of outflow activity from T-Tauri stars, as compared to optical forbidden emission. Astron. Astrophys. 417, 247-261 (2004).

18. Takami, M., Bailey, J. \& Chrysostomou, A. A spectro-astrometric study of southern pre-main sequence stars. Binaries, outflows, and disc structure down to AU scales. Astron. Astrophys. 397, 675-691 (2003).

19. Bailey, J. Detection of pre-main-sequence binaries using spectro-astrometry. Mon. Not. R. Astron. Soc. 301, 161-167 (1998)

Supplementary Information is linked to the online version of the paper at www.nature.com/nature.

Acknowledgements This work was supported in part by Science Foundation Ireland and the JETSET Marie Curie research training network.

Author Information Reprints and permissions information is available at npg.nature.com/reprintsandpermissions. The authors declare no competing financial interests. Correspondence and requests for materials should be addressed to E.T.W. (ewhelan@cp.dias.ie). 